\title{
On the Problem of Choosing Subgroups of Clifford Algebras for Applications in Funda- mental Physics
}

\author{
Robert Arnott Wilson*(1)
}

\author{
Communicated by Vladislav Kravchenko
}

\begin{abstract}
Clifford algebras are used for constructing spin groups, and are therefore of particular importance in the theory of quantum mechanics. An algebraist's perspective on the many subgroups and subalgebras of Clifford algebras may suggest ways in which they might be applied more widely to describe the fundamental properties of matter. I do not claim to build a physical theory on top of the fundamental algebra, and my suggestions for possible physical interpretations are indicative only, and may not work. Nevertheless, both the existence of three generations of fermions and the symmetry-breaking of the weak interaction seem to emerge naturally from an extension of the Dirac algebra from complex numbers to quaternions.
\end{abstract}

\section{Introduction}

\subsection{Clifford Algebras in the Standard Model}

The spin-type groups used in physics $[17,47,49]$ include not only the ordinary spin group $\operatorname{Spin}(3) \cong S U(2)$ and the relativistic [6] spin group $\operatorname{Spin}(3,1) \cong$ $S L(2, \mathbb{C})$, but also the gauge groups $\operatorname{Spin}(2) \cong U(1)$ of quantum electrodynamics [11] and $\operatorname{Spin}(3) \cong S U(2)$ of the weak interaction [39]. Each of these groups arises from two different Clifford algebras [30], so that already we have at least 6 distinct Clifford algebras in the picture, namely $C \ell(2,0), C \ell(0,2)$, $C \ell(3,0), C \ell(0,3), C \ell(3,1)$ and $C \ell(1,3)$.

The Dirac algebra, as used in the Feynman calculus in the standard model of particle physics, is a complex version of the real algebra $C \ell(1,3)$ generated by the Dirac gamma matrices $\gamma_{1}, \gamma_{2}, \gamma_{3}$ and $\gamma_{0}$, whose essential properties are that they anti-commute with each other, and square to -1 ,

\footnotetext{
${ }^{*}$ Corresponding author
} 
$-1,-1$ and +1 respectively. Instead of using the scalar $i$ as a fifth generator, it is conventional (or at least instructive) to use the matrix $\gamma_{5}:=i \gamma_{0} \gamma_{1} \gamma_{2} \gamma_{3}$, which anti-commutes with the first four gamma matrices and squares to +1 .

With respect to these five gamma matrices as generators, therefore, the Dirac algebra has the structure of $C \ell(2,3)$, that is the Clifford algebra of anti-de Sitter space [23], in contrast to its usual interpretation as the complex Clifford algebra of Minkowski space, $\mathbb{C} \ell(3,1)=\mathbb{C} \ell(1,3)$. Another reasonable set of generators is

$$
\gamma_{5}, i \gamma_{1}, i \gamma_{2}, i \gamma_{3}, i \gamma_{0}
$$

which gives a structure $C \ell(4,1)$ on de Sitter space, and a third possibility is

$$
\gamma_{1}, \gamma_{2}, \gamma_{3}, i \gamma_{0}, i \gamma_{5}
$$

which gives a structure of $C \ell(0,5)$. All these algebras are isomorphic (as algebras) to the algebra of all $4 \times 4$ complex matrices.

The same physical information can be packaged into slightly different mathematics by reversing the signature of the Clifford algebra. We obtain $C \ell(3,2)$, which consists of two copies of the algebra of $4 \times 4$ real matrices, and $C \ell(5,0)$ and $C \ell(1,4)$, both of which consist of two copies of $2 \times 2$ quaternion matrices. Since these are not isomorphic as algebras to the Dirac algebra, they are less likely to be useful here, but should not be ruled out of consideration entirely.

\subsection{Clifford Algebras Beyond the Standard Model}

Larger Clifford algebras have also been suggested [12] for purposes such as including the strong force [15] gauge group $S U(3)$, although $S U(3)$ is not itself a spin group. But $S U(3)$ is a subgroup of $S O(6)$, and by the Klein correspondence $\operatorname{Spin}(6) \cong S U(4)$, which contains $S U(3)$ as a subgroup. Hence this approach may also be useful in extending from the standard model to a 'four-colour' model based on $S U(4)$, such as the Pati-Salam model [2,25]. Similarly, Penrose's twistor theory $[1,26]$ is based on $\operatorname{Spin}(4,2) \cong S U(2,2)$, which can be constructed via the Clifford algebra $C \ell(4,2)$ or $C \ell(2,4)$. In fact, the algebras $C \ell(3,3), C \ell(0,6)$ and $C \ell(4,2)$ are all isomorphic to the algebra of $8 \times 8$ real matrices, so all three approaches can be pursued simultaneously if desired. The reversed signatures $C \ell(6,0)$ and $C \ell(2,4)$ are both isomorphic to the algebra of $4 \times 4$ quaternion matrices, as are $C \ell(5,1)$ and $C \ell(1,5)$, so that these provide an alternative approach for those who appreciate the virtues of using quaternions [24].

By now, I have mentioned 19 distinct real Clifford algebras, and it is obvious that some clear mathematical principles are going to be necessary in order to make good choices for which ones to use in which physical contexts. The main principle I want to discuss in this paper is the principle of choosing a particular Clifford algebra structure from the many that are in general available in a suitable abstract algebra. This is a mathematical symmetrybreaking principle, which may or may not have anything to do with the physical process of 'spontaneous symmetry-breaking' that is usually invoked to explain the structure of the fundamental forces. 
Choosing a Clifford algebra structure is equivalent to choosing a particular subgroup of the algebra to call 'the' spin group, so that one can also approach the problem from the point of view of studying subgroups [32-34] of the Clifford algebra. It is intimately connected with the question of choosing a particular real form of the Clifford algebra and the associated spin group. In the physics literature, Clifford algebras are usually complexified, so that the issue of choosing a real form does not arise. I hope to show that the difference between the different real forms has real consequences for physics, and is therefore worth paying attention to.

A number of recent papers, such as [35], have sought to use the complex Clifford algebra $\mathbb{C} \ell(6)$ to combine the electroweak part of the standard model, usually implemented in the Dirac algebra $\mathbb{C} \ell(4)$, with the strong part of the model, usually implemented in $S U(3)$. As far as I am aware, these papers aim at re-packaging the standard model in a unified notation, rather than going beyond the standard model. The latter aim is generally attempted only with a larger algebra [37]. There is also a strong link between this Clifford algebra and the octonions, which is exploited by Furey [13,14] in particular to build octonionic models that unify the strong $S U(3)$ with the rest of the standard model in a single algebraic framework. The octonions provide an elegant construction of the spin groups $\operatorname{Spin}(8)$ and $\operatorname{Spin}(4,4)$, so can be used instead of Clifford algebras for this purpose.

\subsection{Aims}

My concern in this paper is rather different from all of the above, namely to try to deal with some issues that the standard model does not address, without going outside the Clifford algebra framework. In particular, I want to address the existence of three generations of fermions, and some aspects of symmetry-breaking and the mixing between the different forces. This paper is one of a series in which I look at some of the fundamental problems of physics from a group-theoretical point of view. An overview is given in [40], and some alternative approaches based on finite groups are described in [41,42].

\section{Symmetry-Breaking}

\subsection{Examples}

Every Clifford algebra is either a full matrix algebra over the real numbers, complex numbers or quaternions, or the direct sum of two such algebras, isomorphic to each other. Moreover, the algebra contains much larger groups than the spin group it was designed to construct. Restricting from the large group of all invertible elements of the Clifford algebra, to the spin group, is a process of breaking a large symmetry group down to a small one. For example, $C \ell(0,2)$ is a quaternion algebra, so contains not only $\operatorname{Spin}(2) \cong U(1)$, but also a copy of $S U(2) \cong \operatorname{Spin}(3)$. Another example that I hope to show is relevant to physics is $C \ell(3,1)$, that is a $4 \times 4$ real matrix algebra, so contains not only $\operatorname{Spin}(3,1) \cong S L(2, \mathbb{C})$ but also a copy of $S L(4, \mathbb{R}) \cong \operatorname{Spin}(3,3)$.

In both these cases, the Clifford algebra breaks the symmetry. Thus $C \ell(0,2)$ breaks the symmetry of $S U(2)$ by choosing a particular subgroup 
$U(1)$ to call $\operatorname{Spin}(2)$. This example may be relevant to the symmetry-breaking of the weak gauge group $S U(2)$, that distinguishes between the neutral $Z$ boson and the two charged $W$ bosons. Similarly, $C \ell(3,1)$ breaks the symmetry of $S L(4, \mathbb{R})$ by choosing a particular copy of $S L(2, \mathbb{C})$ to call $\operatorname{Spin}(3,1)$. In the Lie algebra context, this example is quite familiar to physicists, and has been used in a recent proposed $E_{8}$ model [4] for the breaking of symmetry between the three generations of fermions. My point, however, is that this symmetrybreaking arises more naturally in the context of small Clifford algebras than in the context of large Lie algebras.

To see this operation of symmetry-breaking in a bigger context, we can use the Clifford algebra $C \ell(3,3)$ to break the symmetry of the matrix group $S L(8, \mathbb{R})$ down to $\operatorname{Spin}(3,3) \cong S L(4, \mathbb{R})$. Then we can use $C \ell(3,1)$ to break $S L(4, \mathbb{R})$ symmetry down to $\operatorname{Spin}(3,1) \cong S L(2, \mathbb{C})$. The latter lies in both $C \ell(3,0)$ and $C \ell(1,2)$, which can be used to break the symmetry to either of the groups $\operatorname{Spin}(3) \cong S U(2)$ or $\operatorname{Spin}(1,2) \cong S L(2, \mathbb{R})$, according to preference.

If further symmetry-breaking is required, one can use $C \ell(0,2)$ to break $S U(2)$ down to $U(1)$, and $C \ell(2,0)$ to break $S L(2, \mathbb{R})$ down to $U(1)$, or even use $C \ell(1,1)$ to break $S L(2, \mathbb{R})$ down to $G L(1, \mathbb{R})$. These chains of symmetrybreaking should be sufficient to deal with the main examples of symmetrybreaking in the standard model. The chain down through $C \ell(3,1), C \ell(3,0)$ and $C \ell(2,0)$ looks particularly promising.

\subsection{A Quaternionic Dirac Algebra}

One of many ways to construct $C \ell(3,3)$ is to extend the complex numbers in the Dirac algebra to the quaternions. This is not just a matter of extending the $4 \times 4$ complex matrices to $4 \times 4$ quaternion matrices, but of adjoining quaternions $i, j, k$ that commute with the four Dirac matrices $\gamma_{1}, \gamma_{2}, \gamma_{3}$ and $\gamma_{0}$. This process requires $8 \times 8$ real matrices rather than $4 \times 4$ quaternion matrices, since $j$ and $k$ must anti-commute with $\gamma_{5}$. Details are given in Sect. 4.2 .

To obtain the required anti-commuting generators we can take

$$
i \gamma_{1}, i \gamma_{2}, i \gamma_{3}, i \gamma_{0}, j, k
$$

which exhibits the structure of $C \ell(3,3)$. Some alternative sets of generators for $C \ell(3,3)$ are

$$
\begin{aligned}
& \gamma_{1}, \gamma_{2}, \gamma_{3}, \gamma_{0}, j \gamma_{5}, k \gamma_{5} \\
& \gamma_{1}, \gamma_{2}, \gamma_{3}, \gamma_{5}, j \gamma_{5}, k \gamma_{5}
\end{aligned}
$$

It is not immediately obvious which of these choices will be most useful for physical applications. But the mathematical structure of $C \ell(3,0)$ is quite different from that of $C \ell(0,3)$, so the choice between $\gamma_{1}, \gamma_{2}, \gamma_{3}$ generating $C \ell(0,3)$ and $i \gamma_{1}, i \gamma_{2}, i \gamma_{3}$ generating $C \ell(3,0)$ is likely to be significant. The other important choice here is between using $\gamma_{0}$ or $\gamma_{5}$ as pseudoscalar (the product of all the generators).

As noted above, the signatures $(0,6)$ and $(4,2)$ are available in the same abstract algebra. Explicit matrices in the former case are given by Fairlie and 
Manogue [10]. We may take the following two sets of generators, both with pseudoscalar $i \gamma_{5}$ :

$$
\begin{gathered}
\gamma_{1}, \gamma_{2}, \gamma_{3}, i \gamma_{0}, j \gamma_{0}, k \gamma_{0} \\
i \gamma_{1}, i \gamma_{2}, i \gamma_{3}, \gamma_{0}, j \gamma_{0} \gamma_{5}, k \gamma_{0} \gamma_{5}
\end{gathered}
$$

We therefore have a choice of three distinct spin groups

$$
\operatorname{Spin}(3,3) \cong S L(4, \mathbb{R}), \quad \operatorname{Spin}(0,6) \cong S U(4), \quad \operatorname{Spin}(4,2) \cong S U(2,2)
$$

but only the first of these is suitable for further symmetry-breaking with a smaller Clifford algebra. Both $C \ell(3,1)$ and $C \ell(2,2)$ are available for this purpose, and although the former is the 'obvious' one, the latter may also be useful. The remaining spin group

$$
\operatorname{Spin}(5,1) \cong S L(2, \mathbb{H})
$$

can similarly be used as input to further symmetry-breaking using any of $C \ell(4,0), C \ell(1,3)$ or $C \ell(0,4)$.

For now I will stick as closely as possible to the standard model, and work with $C \ell(3,3)$ as an algebra of $8 \times 8$ real matrices, with $C \ell(3,1)$ used for symmetry-breaking of $\operatorname{Spin}(3,3)$. I have suggested three possible generating sets, two of which have pseudoscalar $\gamma_{5}$, while the third has pseudoscalar $\gamma_{0}$. The choice between these options must be made on the basis of subgroups other than the spin group itself.

\section{Subgroups}

\subsection{Electroweak Gauge Groups}

In the standard model Dirac algebra $C \ell(2,3)$, the modelling of electro-weak interactions uses the 2-dimensional complex subalgebra spanned by 1 and $\gamma_{5}$. This permits the implementation of two copies of $U(1)$, generated as Lie groups by $i$ and $i \gamma_{5}$. In other words, only a subgroup $U(1)$ of the weak gauge group $S U(2)$ appears explicitly in the Dirac algebra. The full $S U(2)$ can only be implemented by extending to the quaternionic Dirac algebra $C \ell(3,3)$ described in Sect. 2.2. The Coleman-Mandula theorem [5] implies that the gauge group must lie in the centralizer algebra of the relativistic spin group $S L(2, \mathbb{C})$, generated by even products of the original four gamma matrices. This centralizer algebra is generated by $j, k$ and $\gamma_{5}$, and is therefore isomorphic to $C \ell(1,2)$, and thereby to the algebra of $2 \times 2$ complex matrices.

By choosing instead the generators $j \gamma_{5}, k \gamma_{5}$ and $\gamma_{5}$ we can identify this algebra also as $C \ell(3,0)$. Which generators should we choose? The four rotations are $i, j, k$ and $i \gamma_{5}$, and the three boosts are $\gamma_{5}, j \gamma_{5}$ and $k \gamma_{5}$. Whatever happens, the symmetry is visibly broken, but we have a choice of which bits of symmetry to keep, and which to abandon. This is largely a matter of physical interpretation, rather than mathematics, so it is not appropriate to try to make such a decision here. What is clear, however, is that the four rotations give a group $U(1)$ generated by $i \gamma_{5}$, and a group $S U(2)$ generated by $i, j, k$, and that these groups commute with each other, and generate a group $U(2)$. 
This is exactly the formalism that is normally used to describe the unification of quantum electrodynamics (QED) with the weak force.

Thus consideration of the electro-weak interactions suggests that the third of the three proposed sets of generators for $C \ell(3,3)$ is likely to be the most useful. There appear to be some minor technical differences between what we see here and what appears in the Feynman calculus, in that the roles of $i$ and $i \gamma_{5}$ appear to have been interchanged. Since this can be adjusted by applying complex conjugation on half of the spinor, I suspect this is not a serious issue. Indeed, this difference may be more apparent than real: it may well be possible to take the Feynman calculus for one generation exactly as it is, and then to use $j \gamma_{5}$ and $k \gamma_{5}$ for the second and third generations, in place of $\gamma_{5}$.

There is complete symmetry amongst these three elements of the Clifford algebra, that is slightly obscured by the conventional notation. If we write them instead as $i \gamma_{0} \gamma_{1} \gamma_{2} \gamma_{3},-k \gamma_{0} \gamma_{1} \gamma_{2} \gamma_{3}$ and $j \gamma_{0} \gamma_{1} \gamma_{2} \gamma_{3}$, the symmetry becomes obvious. It may therefore be possible to use these three elements of the larger algebra to define three different copies of the Dirac algebra, suitable for modelling the three generations of fermions. Since the symmetry group here is the symmetry group of the quaternions, if we choose $i, j, k$ as a basis suitable for the three generations of electrons, then we can change basis arbitrarily in order to implement another basis suitable for the quarks. With this choice of notation, the electron generation symmetry can be expressed explicitly by the element $(-1+i+j+k) / 2$.

There is also no clearcut answer to the question whether we should use compact $S U(2)$ or split $S L(2, \mathbb{R})$ or complex $S L(2, \mathbb{C})$ as the gauge group. This ambiguity also exists in the standard model, so again, there should not be a problem with this. Indeed, the groups themselves play a rather minor role in the theory: it is the Clifford algebra where all the calculations take place. It is clear, I think, that electro-weak mixing can be expressed mathematically, not as a process of 'mixing' groups together, or mixing Lie algebras together, but as a process of choosing bases for the matrix algebra to turn it into one or more Clifford algebras. This viewpoint has some consequences for how to think about gauge groups in the general case. Although they may sometimes appear in the formalism as groups or Lie algebras, it is certainly possible that these mathematical objects are secondary to a primary manifestation as a Clifford algebra.

\subsection{Spin Groups}

The ordinary spin group $\operatorname{Spin}(3) \cong S U(2)$ is generated as a Lie group by $\gamma_{1} \gamma_{2}, \gamma_{2} \gamma_{3}$ and $\gamma_{3} \gamma_{1}$. The corresponding algebra can be regarded either as the even part of $C \ell(3,0)$, generated by $i \gamma_{1}, i \gamma_{2}, i \gamma_{3}$, or as the even part of $C \ell(0,3)$, generated by $\gamma_{1}, \gamma_{2}, \gamma_{3}$. The algebra $C \ell(3,0)$ is isomorphic to the algebra of all $2 \times 2$ complex matrices, and therefore contains a subgroup $S L(2, \mathbb{C})$ that is isomorphic to, but not equal to, the relativistic spin group. I do not know if this copy of the group $S L(2, \mathbb{C})$ has any reasonable physical interpretation. 
The algebra $C \ell(0,3)$, on the other hand, is the direct sum of two copies of the quaternion algebra. Generators for these two copies may be taken as:

$$
\begin{gathered}
\gamma_{1}+\gamma_{2} \gamma_{3}, \gamma_{2}+\gamma_{3} \gamma_{1}, \gamma_{3}+\gamma_{1} \gamma_{2} \\
\gamma_{1}+\gamma_{3} \gamma_{2}, \gamma_{2}+\gamma_{1} \gamma_{3}, \gamma_{3}+\gamma_{2} \gamma_{1}
\end{gathered}
$$

They therefore have a fairly obvious interpretation as left-handed and righthanded spins. Exponentiating these generators in the usual way we obtain two copies of $S U(2)$, one left-handed and one right-handed. These two copies of $S U(2)$ intersect trivially, and therefore they generate a copy of $\operatorname{Spin}(4)$.

The same effect is achieved in the standard model using $C \ell(4,0)$ generated by $\gamma_{0}, i \gamma_{1}, i \gamma_{2}, i \gamma_{3}$, with pseudoscalar element $\gamma_{5}$. Here the full Clifford algebra is a $2 \times 2$ quaternion matrix algebra, and the even part is the sum of two quaternion algebras, which can be separated by the projections with the idempotents $\left(1 \pm \gamma_{5}\right) / 2$. The generators can be taken as

$$
\begin{gathered}
\gamma_{1} \gamma_{2}+i \gamma_{0} \gamma_{3}, \gamma_{2} \gamma_{3}+i \gamma_{0} \gamma_{1}, \gamma_{3} \gamma_{1}+i \gamma_{0} \gamma_{2} \\
\gamma_{1} \gamma_{2}-i \gamma_{0} \gamma_{3}, \gamma_{2} \gamma_{3}-i \gamma_{0} \gamma_{1}, \gamma_{3} \gamma_{1}-i \gamma_{0} \gamma_{2}
\end{gathered}
$$

The obvious question that occurs to an algebraist [38] at this point is whether the extra complications in the standard model are actually necessary, if the same effect can be achieved with the simpler mathematical machinery presented above? That is, can we take the same spin terms as always, $\gamma_{1} \gamma_{2}$ and so on, but project with $\left(1 \pm \gamma_{1} \gamma_{2} \gamma_{3}\right) / 2$ without the extra factor of $i \gamma_{0}$ ? I do not pretend to provide an answer to this, I merely ask the question.

The relativistic spin group $\operatorname{Spin}(3,1) \cong S L(2, \mathbb{C})$ can be obtained either from the even part of $C \ell(3,1)$, generated by $i \gamma_{\mu}$ for $\mu=0,1,2,3$, or from the even part of $C \ell(1,3)$, generated by the $\gamma_{\mu}$ themselves. The algebra $C \ell(3,1)$ is isomorphic to the $4 \times 4$ real matrix algebra, while $C \ell(1,3)$ is isomorphic to the $2 \times 2$ quaternion matrix algebra, and the even part in both cases is isomorphic to the $2 \times 2$ complex matrix algebra. In both cases the pseudoscalar is $\gamma_{0} \gamma_{1} \gamma_{2} \gamma_{3}$, which squares to -1 . Hence there are no projections onto left-handed and right-handed spins as such, but in the standard model this distinction is made instead by complex conjugation. This is done by extending the Clifford algebra either from $C \ell(3,1)$ to $C \ell(4,1)$, or from $C \ell(1,3)$ to $C \ell(2,3)$. Or, equivalently, complexifying the Clifford algebra.

So again, the question that occurs to an algebraist is, is this extension really necessary, or is there already enough information in the odd part of $C \ell(3,1)$ or $C \ell(1,3)$ ? In both cases, the Clifford algebra provides the necessary symmetry-breaking, either by choosing a copy of the complex numbers inside the quaternions, or by imposing a complex 2-space structure onto a real 4 -space. The discussion in this section suggests that the algebra $C \ell(1,3)$ provides a closer link to experimental properties of left-handed and right-handed spin, while the standard model is somewhat ambivalent.

\subsection{The Mass Term in the Dirac Equation}

In the standard model Clifford algebra, whether it is regarded as $C \ell(4,1)$ or $C \ell(2,3)$ or $C \ell(0,5)$, the pseudoscalar term is always $i$. This pseudoscalar is conventionally used for the mass term in the Dirac equation. But it may not 
be the most natural choice. The Dirac equation was modelled on a factorisation, into two linear factors, of Einstein's equation

$$
m^{2} c^{2}=-p^{2}+E^{2} / c^{2},
$$

in that particular form, rather than, for example,

$$
E^{2} / c^{2}=m^{2} c^{2}+p^{2} .
$$

If the mass term is a scalar, then any re-arrangement of the equation introduces unphysical cross-terms in the squaring process, so is not possible. But the symmetrical form

$$
m^{2} c^{2}+p^{2}-E^{2} / c^{2}=0
$$

suggests that we should really be looking at a group $S O(4,1)$, and therefore the Clifford algebra $C \ell(4,1)$, with generators $i \gamma_{\mu}$ for the energy and momentum terms, and therefore $\gamma_{5}$ for the mass term. This is consistent with the earlier observation (Sect. 3.1) that extending to three generations of fermions seems to force the roles of $i$ and $i \gamma_{5}$ to be interchanged.

Indeed, if we want to extend to a larger Clifford algebra in order to incorporate the three generations of fermions, then $i$ is no longer a natural choice for pseudoscalar. The discussion so far has suggested either $\gamma_{0}$ or $\gamma_{5}$ as pseudoscalar, with a preference (see Sect. 3.1) for $\gamma_{0}$. The question really is whether (rest) mass $\left(\gamma_{5}\right)$ or (total) energy $\left(\gamma_{0}\right)$ is a better choice for pseudoscalar. One has to make a choice on physical, not mathematical, grounds. Experiment makes it clear that mass is not conserved in the weak interaction, while the whole of physics relies on the principle of conservation of energy. This suggests that taking $\gamma_{0}$ as the pseudoscalar may ultimately result in a more fundamental theory.

The current suggestion for generators for the quaternionic Dirac algebra is as follows, in two different notations, the second, more cumbersome, notation exhibiting the symmetry between the three generations of fermions:

$$
\begin{aligned}
& \gamma_{1}, \gamma_{2}, \gamma_{3}, \gamma_{5}, j \gamma_{5}, k \gamma_{5} ; \\
& \gamma_{1}, \gamma_{2}, \gamma_{3}, i \gamma_{0} \gamma_{1} \gamma_{2} \gamma_{3}, j \gamma_{0} \gamma_{1} \gamma_{2} \gamma_{3}, k \gamma_{0} \gamma_{1} \gamma_{2} \gamma_{3} .
\end{aligned}
$$

This version has pseudoscalar $\gamma_{0}$, which contrasts with the pseudoscalar $i \gamma_{5}$ in the standard model Dirac algebra $\mathbb{C} \ell(3,1)$. This change of pseudoscalar has the effect that the relativistic spin group $S L(2, \mathbb{C})$, defined in terms of $\gamma_{1}, \gamma_{2}, \gamma_{3}, \gamma_{0}$, does not lie inside the copy of $\operatorname{Spin}(3,3)$ defined by this $C \ell(3,3)$ structure.

Instead, inside $\operatorname{Spin}(3,3)$ there are three copies of $S L(2, \mathbb{C})$, one for each generation, each acting on the 6 -vector representation of $S O(3,3)$ as a Minkowski 4-vector plus two scalars. In particular, these Minkowski spaces cannot be interpreted as spacetime or 4-momentum representations. This is in contrast to many approaches, such as [4], in which the identification of two distinct copies of $S L(2, \mathbb{C})$ entails a counter-intuitive hypothesis of 3 dimensional time in order to incorporate the three generations. I have no need of this hypothesis, since I distinguish between four explicit copies of $S L(2, \mathbb{C})$ rather than three, and thereby distinguish between one dimension of energy/time and three dimensions of generations/mass. 
Now it is worth pointing out that $S O(3,3)$ contains three distinct conjugacy classes of subgroups isomorphic to $S O(3,1)$, one of which contains the three 'generation' copies just discussed. There is another class obtained by fixing a direction of spin, for example by choosing just one of $\gamma_{1}, \gamma_{2}, \gamma_{3}$ to lie in the associated Clifford algebra. These also have Minkowski 4-space representations in the 6 -vector, but with the opposite signature. The third class is quite different, as it has a Minkowski 4-space representation in the spinor representation of $\operatorname{Spin}(3,3)$ instead, and a complex 3-vector in the 6-vector representation.

As far as I am aware, this third class of $S O(3,1)$ is not used in particle physics, although in general relativity it is identified with the Lorentz group. In other words, the two subjects do not agree about which mathematical copy of $S O(3,1)$ to call the Lorentz group. I believe this may be one important source of difficulty in unification. Current theories only accept the existence of one group $S O(3,1)$ in physics, whereas a careful mathematical analysis suggests there are many, and they need to be carefully distinguished. This separation of four physically different copies of $S O(3,1)$ is something I have not seen in other models, and it may have some important consequences, which are discussed further in Sects. 5.1 and 5.4.

At this point, however, we are not concerned with unification. I have made a proposal for embedding the electro-weak gauge groups and the spin groups into the quaternionic Dirac algebra $C \ell(3,3)$, and the stage is set for attempting to reproduce the rest of the standard model, with three generations of fermions, and including the mixing angles and other parameters. Obtaining the values of the parameters is not a matter of algebra, but obtaining a classification of the parameters is.

\section{The Structure of the Clifford Algebra}

\subsection{Undetermined Parameters}

There are some parameters within the algebra $C \ell(3,0)$, that describes electroweak unification. These parameters include one that describes the breaking of the symmetry within $S U(2)$, and three that describe how the pseudoscalar $U(1)$ mixes with the three dimensions of $S U(2)$.

The remaining parameters describe how $C \ell(3,0)$ relates to $C \ell(0,3)$. The latter has 8 dimensions in total, but under rotation symmetries of space these reduce to 4 different types, in degrees $0,1,2$ and 3 . The degree 0 is the identity element of the algebra, so cannot mix with anything. Similarly, $C \ell(3,0)$ has 8 dimensions, and since the symmetry is broken, all 7 non-identity elements participate individually in the mixing. Hence there are $3 \times 7=21$ more parameters, making a total of $4+21$, exactly the number required for the standard model, according to some counts at least. The 25 parameters consist of $5 \times 3$ mass terms, for 12 fermions and 3 bosons, $3+1$ mixing angles each from the Cabibbo-Kobayashi-Maskawa (CKM) matrix [3,20] and the Pontecorvo-Maki-Nakagawa-Sakata (PMNS) matrix $[22,29]$, plus the finestructure constant and the strong coupling constant. 
The main group of 21 parameters splits under electroweak symmetries, according to my suggestion, into 3 sets of $3+3+1$, of which one may contain lepton masses, and one quark masses, leaving the other to contain $3+3+1$ of the $3+1+3+1$ mixing angles. The other two scalars here, I suggest, might be the fine-structure constant (to go with the lepton masses) and the strong coupling constant (to go with the quark masses). That leaves the electroweak group of four parameters as the three boson masses (Higgs, $Z$ and $W$ ), together with the last mixing angle, which may be the CP-violating phase in the CKM matrix. This proposed allocation of parameters may not be exactly correct, of course, but it shows that the overall pattern of parameters matches closely to the standard model.

In a little more detail, we can label the $3 \times 7$ parameters with products of $\gamma_{3}, \gamma_{1} \gamma_{2}, \gamma_{1} \gamma_{2} \gamma_{3}$ with $i, j, k, \gamma_{5}, i \gamma_{5}, j \gamma_{5}, k \gamma_{5}$, and the remaining four with the products of $\gamma_{5}$ with $1, i, j, k$. In the main block of $3 \times 7$ parameters, the $i, j, k$ symmetry is a generation symmetry for fermions, while in the remaining four it represents the broken symmetry $1, k, j$ of the weak interaction, since there is a hidden multiplication by $i$ in the theory.

\subsection{A Quaternionic Notation}

Since the notation inherited from Dirac obscures some of the symmetries, in particular the generation symmetry, I suggest an alternative notation. The algebra $C \ell(1,3)$ is the algebra of $2 \times 2$ quaternion matrices, so is the tensor product of quaternions with $2 \times 2$ real matrices. Let us take $i^{\prime}, j^{\prime}, k^{\prime}$ for these quaternions, and represent them as $4 \times 4$ matrices acting on quaternions by left-multiplication by $-i,-j,-k$, commuting with right-multiplications by $i, j, k$ already defined. Then take $2 \times 2$ real matrices

$$
I:=\left(\begin{array}{cc}
0 & 1 \\
-1 & 0
\end{array}\right), \quad J:=\left(\begin{array}{ll}
0 & 1 \\
1 & 0
\end{array}\right), \quad K:=I J=\left(\begin{array}{cc}
1 & 0 \\
0 & -1
\end{array}\right) .
$$

Then we can take Dirac matrices as generators, defined as follows:

$$
\gamma_{1}:=J i^{\prime}, \quad \gamma_{2}:=J j^{\prime}, \quad \gamma_{3}:=J k^{\prime}, \quad \gamma_{0}:=K .
$$

Our chosen copy of $C \ell(0,3)$ is then generated by $J i^{\prime}, J j^{\prime}, J k^{\prime}$, and our chosen copy of $C \ell(3,0)$ by $I i, I j, I k$. Space symmetry is described by $i^{\prime}, j^{\prime}, k^{\prime}$, and generation symmetry by $i, j, k$. The pseudoscalar elements of these two subalgebras and the whole algebra are

$$
J=-\gamma_{1} \gamma_{2} \gamma_{3}, \quad I=i \gamma_{5}, \quad K=\gamma_{0}
$$

respectively. One then sees the breaking of symmetry between the three generations in the choice of $\gamma_{5}=-I i$ in the standard model.

The even part of the algebra corresponds to the diagonal matrices 1 and $K$, while the odd part corresponds to the off-diagonal matrices $I$ and $J$. The ordinary spin group $S U(2)$ is generated by $i^{\prime}, j^{\prime}, k^{\prime}$, and extends to the relativistic spin group generated by $I i^{\prime}, I j^{\prime}, I k^{\prime}$. The weak gauge group appears either as $S U(2)$ generated by $i, j, k$, or as $S L(2, \mathbb{R})$ generated by $i, I j, I k$, with a commuting copy of $U(1)$ generated by $I$. In particular, we have separated two concepts of complexification: one with $I$ and one with $i$. 
These two are mixed together in the standard model, in a way that depends on the choice of the first generation of fermions as special.

To describe the 25 parameters, we must choose an arbitrary direction in space, say the $k^{\prime}$ direction, corresponding to the conventional choice of the $z$ direction in which to measure spin. Then we must mix the three elements $J, k^{\prime}, J k^{\prime}$ in $C \ell(0,3)$ with the seven elements $i, j, k, I, I i, I j, I k$ in $C \ell(3,0)$. The remaining four parameters are perhaps best thought of as lying in $i, I i, I j, I k$, and the whole system of 25 parameters can then be arranged as follows:

\begin{tabular}{|ccc|ccc|c}
$i$ & & & $I i$ & $I j$ & $I k$ & \\
\hline$J i$ & $J j$ & $J k$ & $K i$ & $K j$ & $K k$ & $K$ \\
$k^{\prime} i$ & $k^{\prime} j$ & $k^{\prime} k$ & $I k^{\prime} i$ & $I k^{\prime} j$ & $I k^{\prime} k$ & $I k^{\prime}$ \\
$J k^{\prime} i$ & $J k^{\prime} j$ & $J k^{\prime} k$ & $K k^{\prime} i$ & $K k^{\prime} j$ & $K k^{\prime} k$ & $K k^{\prime}$
\end{tabular}

The second row of parameters does not have a momentum direction $k^{\prime}$, and the first six of them are rotations, and contain generation labels $i, j, k$, so could reasonably be interpreted as the mixing angles between the three generations in the CKM and PMNS matrices. At a guess, $J i, J j, J k$ lie in the CKM matrix, and $K i, K j, K k$ in the PMNS matrix, although speculation on such details is rather pointless at this stage. The first six elements in each of the bottom two rows have momentum and generation labels, and 9 of them are boosts, so could reasonably be interpreted as 9 of the 12 fundamental fermion masses. The remaining 3 are rotations, so perhaps represent the so far undetected neutrino masses, or some other generation-dependent property.

Of the remaining 7 parameters, two are rotations, and I tentatively suggest $i$ to hold the CP-violating phase of the CKM matrix, and $K k^{\prime}$ to hold the $\mathrm{CP}$-violating phase of the PMNS matrix. This leaves five boosts, $K, I k^{\prime}, I i$, $I j$ and $I k$, to hold the remaining three masses and two coupling constants. A reasonable guess might be that those involving $I$ are electroweak parameters, and $K$ is a strong force parameter, therefore the strong coupling constant. Perhaps $I k^{\prime}$ is the fine structure constant, and the remaining three the masses of the Higgs, $Z$ and $W$ bosons. But electroweak symmetry-breaking may significantly complicate this picture.

\subsection{Even and Odd}

The even part of $C \ell(3,3)$ consists of degrees $0,2,4$ and 6 , with dimensions 1 , 15,15 and 1 respectively. Bases are as follows, arranged with degrees 0 and 4 in the first block, and degrees 2 and 6 in the second:

\begin{tabular}{|c|c|c|c|c|}
\hline 1 & $K i K j K k$ & $K$ & $i$ & $k$ \\
\hline$K i^{\prime}$ & $i^{\prime} i \quad i^{\prime} j \quad i^{\prime} k$ & $i^{\prime}$ & $K i^{\prime} i \quad K i^{\prime} j$ & $K i^{\prime} k$ \\
\hline$K j^{\prime}$ & $j^{\prime} i j^{\prime} j j^{\prime} k^{\prime}$ & $j^{\prime}$ & $K j^{\prime} i K j^{\prime} j$ & $K j^{\prime} k$ \\
\hline$K k^{\prime}$ & $k^{\prime} i k^{\prime} j k^{\prime} k$ & $k^{\prime}$ & $K k^{\prime} i K k^{\prime} j$ & $K k^{\prime} k$ \\
\hline
\end{tabular}

Neither block is closed under the Clifford multiplication, but the first block is closed under Jordan multiplication $A B+B A$, and the second is closed under Lie multiplication $A B-B A$. Indeed, the Lie bracket converts the degree 2 part of the algebra into the Lie algebra of $\operatorname{Spin}(3,3)$. Exponentiating the elements of degree 2 therefore gives (the canonical copy of) $\operatorname{Spin}(3,3)$. This 
is the only copy of $\operatorname{Spin}(3,3)$ that preserves the Clifford algebra structure under its action by conjugation.

The pseudoscalar $K$ defines two orthogonal central idempotents, namely $(1 \pm K) / 2$, in the even part of the Clifford algebra. The corresponding projections map onto two 16-dimensional subalgebras, each of which is isomorphic to the full $4 \times 4$ real matrix algebra. Since

$$
(1+K)(1-K)=0
$$

each subalgebra annihilates the other. In particular, each algebra contains a copy of $S L(4, \mathbb{R})$ that acts on only half of the 8-dimensional real spinor. These subgroups may be useful for physics, but it must be stressed that they do not preserve the Clifford algebra structure, and therefore they unavoidably break a great deal of symmetry. They do, however, preserve the distinction between the even and odd parts of the Clifford algebra.

The odd part of the algebra splits into degrees 1,3 and 5 , with dimensions 6, 20 and 6 respectively. Degrees 1 and 5 have bases

$$
\begin{aligned}
& J i^{\prime}, J j^{\prime}, J k^{\prime}, I i, I j, I k ; \\
& I i^{\prime}, I j^{\prime}, I k^{\prime}, J i, J j, J k
\end{aligned}
$$

respectively. As a representation of $\operatorname{Spin}(3,3)$, the degree 3 part splits into a dual pair of 10-dimensional real representations. These are obtained by multiplying the 10 elements

$$
\begin{array}{l|lll}
1 & & & \\
\hline i^{\prime} i & i^{\prime} j & i^{\prime} k \\
j^{\prime} i & j^{\prime} j & j^{\prime} k \\
k^{\prime} i & k^{\prime} j & k^{\prime} k
\end{array}
$$

by $J+I$ and $J-I$ respectively. This splitting corresponds to the splitting into the top-right and bottom-left $4 \times 4$ blocks of the $8 \times 8$ matrices.

Now

$$
(J \pm I)^{2}=0, \quad(J+I)(J-I)=2(1+K), \quad(J-I)(J+I)=2(1-K)
$$

so that each of these two 10-spaces has Clifford product identically zero. Their product in one order gives one projection of the even part of the algebra, and their product in the other order gives the other projection.

\subsection{A Change of Signature?}

The unification of space and time in special relativity mixes momentum (which we might associate with $J i^{\prime}, J j^{\prime}, J k^{\prime}$, in the odd part of the Clifford algebra) with energy (associated with $K$, in the even part). Similarly, the unification of the electroweak interactions mixes $i, j, k$ in the even part with $I$ in the odd part. In other words, both these unifications require groups that extend into the odd part of the Clifford algebra.

This might mean that the Clifford algebra that I have suggested is irrelevant for physics. Or it might mean that the relationship between different Clifford algebra structures may throw some light on the process of symmetrybreaking. In particular, the necessity for using the odd part of the algebra for 
part of the relativistic spin group (conventionally identified with the Lorentz group), and for part of the electro-weak gauge group, suggests the possibility that the strong gauge group $S U(3)$ may also lie partly in the odd part of the algebra.

Alternatively, we may prefer to change our choice of Clifford algebra structure to try to bring the standard model symmetries into the even part of the algebra. Essentially, we have to make $I$ even and $K$ odd. One way to do this is to choose the following generators, given in both notations:

$$
\begin{aligned}
& \gamma_{1}, \gamma_{2}, \gamma_{3}, i \gamma_{0}, j \gamma_{0}, k \gamma_{0} ; \\
& J i^{\prime}, J j^{\prime}, J k^{\prime}, K i, K j, K k
\end{aligned}
$$

This has the effect of changing the signature to $(0,6)$, and the pseudoscalar to $I$ or $i \gamma_{5}$, and changing the spin group to $\operatorname{Spin}(6) \cong S U(4)$.

There are now two obvious ways to collapse the Clifford algebra from a 6 -space to a 4-space, by multiplying together either the first three generators or the last three. Both give copies of $C \ell(1,3)$. One of these has generators equal to the original gamma matrices, and the corresponding spin group $\operatorname{Spin}(1,3)$ is the usual relativistic spin group. The other one has generators $i \gamma_{0} \gamma_{5}, i \gamma_{0}, j \gamma_{0}, k \gamma_{0}$, and the corresponding spin group is generated by

$$
i, j, k, \gamma_{5}, j \gamma_{5}, k \gamma_{5}
$$

Hence it describes electro-weak mixing in exactly the same way as I have already suggested (Sect. 3.1). Both collapsed Clifford algebras have pseudoscalar $i \gamma_{5}$.

There is also an obvious copy of $U(3)$ inside the spin group, generated as a Lie group by the 9 elements

$$
\begin{aligned}
& i+i^{\prime}, I i^{\prime} i, I\left(j^{\prime} k+k^{\prime} j\right), \\
& j+j^{\prime}, I j^{\prime} j, I\left(k^{\prime} i+i^{\prime} k\right), \\
& k+k^{\prime}, I k^{\prime} k, I\left(i^{\prime} j+j^{\prime} i\right) .
\end{aligned}
$$

The scalar copy of $U(1)$ is generated by $I\left(i^{\prime} i+j^{\prime} j+k^{\prime} k\right)$, and $S U(3)$ is generated by the orthogonal complement of this. There are other choices of $S U(3)$, but this is the most symmetrical.

Now comes the problem of interpreting the group $S U(3)$ constructed here. It is hard to support an interpretation as the gauge group of the strong interaction, since the mathematical properties of this copy of $S U(3)$ are not the same as those of the gauge group in the standard model. In particular, it does not commute with the relativistic spin group, generated by

$$
i^{\prime}, j^{\prime}, k^{\prime}, I i^{\prime}, I j^{\prime}, I k^{\prime},
$$

and thereby violates the Coleman-Mandula theorem. Moreover, it does not commute with the weak gauge group generated by $i, j, k$. The only things it does commute with in the even part of the algebra are two copies of the complex numbers, generated by $I$ and $I\left(i^{\prime} i+j^{\prime} j+k^{\prime} k\right)$.

Nevertheless, this copy of $S U(3)$ may repay further study, so let us investigate its action on the Clifford algebra in more detail. We have already 
seen the adjoint representation and a scalar in degree 2 , and the rest of the degree 2 part is a 6 -dimensional real representation on the generators

$$
\begin{aligned}
& i^{\prime}-i, j^{\prime}-j, k^{\prime}-k, \\
& I\left(j^{\prime} k-k^{\prime} j\right), I\left(k^{\prime} i-i^{\prime} k\right), I\left(i^{\prime} j-j^{\prime} i\right) .
\end{aligned}
$$

The latter can be given the structure of a complex 3-space, using the natural scalar $I\left(i^{\prime} i+j^{\prime} j+k^{\prime} k\right)$. The degree 4 part consists of equivalent pieces $8+1+6$, just multiplied by the pseudoscalar $I$. In degrees 1 and 5 we again see copies of the 6-dimensonal real representation, and again there is a natural complex structure defined by $I\left(i^{\prime} i+j^{\prime} j+k^{\prime} k\right)$.

In degree 3 , however, there are two natural complex structures, defined by $I$ and $I\left(i^{\prime} i+j^{\prime} j+k^{\prime} k\right)$. Both give this 20-dimensional space the structure of a complex 10-space, breaking into irreducibles as $1+3+6$. The complex scalars are contained in $J$ and $K$ times $\left(1+i^{\prime} i+j^{\prime} j+k^{\prime} k\right)$. The 3 -space consists of the anti-symmetric part

$$
\begin{aligned}
& J\left(j^{\prime} k-k^{\prime} j\right), J\left(k^{\prime} i-i^{\prime} k\right), J\left(i^{\prime} j-j^{\prime} i\right), \\
& K\left(j^{\prime} k-k^{\prime} j\right), K\left(k^{\prime} i-i^{\prime} k\right), K\left(i^{\prime} j-j^{\prime} i\right),
\end{aligned}
$$

and the 6-space consists of the symmetric part

$$
\begin{aligned}
& J\left(3-i^{\prime} i-j^{\prime} j-k^{\prime} k\right), J\left(i^{\prime} i-j^{\prime} j\right), J\left(j^{\prime} j-k^{\prime} k\right), \\
& J\left(j^{\prime} k+k^{\prime} j\right), J\left(k^{\prime} i+i^{\prime} k\right), J\left(i^{\prime} j+j^{\prime} i\right), \\
& K\left(3-i^{\prime} i-j^{\prime} j-k^{\prime} k\right), K\left(i^{\prime} i-j^{\prime} j\right), K\left(j^{\prime} j-k^{\prime} k\right), \\
& K\left(j^{\prime} k+k^{\prime} j\right), K\left(k^{\prime} i+i^{\prime} k\right), K\left(i^{\prime} j+j^{\prime} i\right) .
\end{aligned}
$$

All of this structure is manifest in the quaternionic Dirac algebra, and therefore must surely have a sensible interpretation in physics. But it seems quite clear that this copy of $S U(3)$ cannot be sensibly interpreted as the gauge group of quantum chromodynamics (QCD). So what is it?

\section{Prospects for Unification}

\subsection{Mass Versus Energy}

Mathematically, the distinction between $C \ell(3,3)$ and $C \ell(0,6)$ is most evident in the distinction between using $I$ or $K$ as pseudoscalar, that is, in physics notation, the distinction between $i \gamma_{5}$ and $\gamma_{0}$. Physically this corresponds to a distinction between symmetry groups for which mass is a scalar, and those for which energy is a scalar. Roughly speaking, mass is a scalar in the theories of special relativity and (classical) electromagnetism, as well as quantum electrodynamics and quantum chromodynamics, while energy is a scalar in general relativity, and in the theory of the weak interaction.

The former (QED and QCD) are the quantum interactions for which the gauge bosons are massless. The gauge groups $U(1)$ and $S U(3)$ therefore embed in the spin group $\operatorname{Spin}(0,6) \cong S U(4)$ which is the subgroup of the Clifford algebra that fixes the mass coordinate. But these groups do not necessarily commute with each other. The group $U(1)$ maps onto a subgroup 
$U(1)$ of $S U(3)$, or more generally of $U(3)$, and this subgroup is defined by a $3 \times 3$ unitary matrix, that appears in the standard model as the CKM matrix.

In the weak interaction, on the other hand, the gauge bosons are massive, so that the gauge group does not act on mass as a scalar, but instead acts on energy as a scalar. Hence the gauge group $S U(2)$ embeds instead in the spin group $\operatorname{Spin}(3,3) \cong S L(4, \mathbb{R})$ that fixes the energy coordinate. Now the Pati-Salam model extends the gauge group $S U(2) \cong S p i n(3)$ acting on three coordinates to $\operatorname{Spin}(4) \cong S U(2) \times S U(2)$

acting on all six. The Clifford algebra formalism identifies the two scalars here, so that the group $\operatorname{Spin}(4)$, which is not a subgroup of $S L(4, \mathbb{R})$, is replaced by $S O(4)$, which is. In other words, the Pati-Salam model in effect uses both Clifford algebra structures $C \ell(0,6)$ supporting $S U(4)$, and $C \ell(3,3)$ supporting $S O(4)$ inside $S L(4, \mathbb{R})$. But the model does not address the issue of symmetry-breaking, which arises from embedding both groups into $S L(8, \mathbb{R})$, by identifying the two Clifford algebras as being two different ways of looking at the same thing.

Similarly, in general relativity, in theory energy is a scalar (conserved, but not necessarily invariant), so that the relevant group is $S L(4, \mathbb{R})$, but most formulations assume that mass is a scalar (both invariant and conserved), which breaks the symmetry down to $S O(3,1)$. Now, in a flat spacetime it is not possible for both mass and energy to be scalars, and therefore general relativity incorporates a curvature of spacetime in order to solve this problem. On the other hand, if one abandons the assumption that mass is a scalar, which we must do in the case of the weak interaction anyway, then it may be possible to construct a version of general relativity in a flat spacetime, with gauge group $S L(4, \mathbb{R})$, in such a way that the curvature of spacetime is replaced by variations in rest mass between different observers, accelerating with respect to each other.

In this way, $C \ell(3,3)$ and the associated group $\operatorname{Spin}(3,3) \cong S L(4, \mathbb{R})$ can potentially describe all the theory that one sees if one assumes that energy is a scalar, including the weak interaction, and a theory of gravity that reduces to general relativity in the limit that rest masses can be treated as constants; while $C \ell(0,6)$ and the associated group $\operatorname{Spin}(0,6) \cong S U(4)$ can describe all the theory one sees if one assumes that mass is a scalar, including electrodynamics and the strong interaction.

Any unified theory that uses a Clifford algebra must reconcile this difference in viewpoint in some way, either by choosing one or the other of mass and energy to be a scalar and allowing the other to vary, or by providing a translation between the two. This fact is already evident in the unification of QED (with scalar mass) and the weak force (with scalar energy). The translation between the two is expressed in the standard model by a symmetry-breaking that arises from treating both mass and energy as scalars simultaneously. This symmetry-breaking is parametrized by an angle, known as the Weinberg angle, which necessarily changes as the relationship between mass and energy changes, as indeed has been observed experimentally.

In terms of both physical principles, and experimental reality, it surely makes more sense to treat energy as a scalar, rather than mass. This is already 
done very effectively in the theory of the weak interaction. But this can only be done by breaking the symmetry groups that rely on mass being a scalar. In particular, this requires breaking the Lorentz symmetry group $S O(3,1)$ down to $S O(3)$, and for QCD the group $S U(3)$ is broken down to nothing using the CKM matrix.

\subsection{The Strong Gauge Group}

If it is really possible to include all the unexplained parameters of the standard model inside the quaternionic Dirac algebra $C \ell(3,3)$, then this algebra must effectively contain the gauge group $S U(3)$ of the strong force as well. But there are some serious mathematical problems with such a proposal, as this Clifford algebra is simply not big enough to contain a commuting product of all the gauge groups and the relativistic spin group. The only copies of $S U(3)$ that are available break the symmetry significantly.

But we have already seen, in the case of electro-weak mixing, that the Clifford algebra imposes a symmetry-breaking that is not obvious in the standard model, so maybe the same is true for the mixing of the strong force with the electroweak forces. The triplet colour symmetry is unobservable, and the only available place to put it is in the triplet $\gamma_{1} \gamma_{2}, \gamma_{2} \gamma_{3}, \gamma_{3} \gamma_{1}$ that is independent of the 25 significant parameters. This unavoidably identifies colour as being essentially the same thing as the direction of spin. The direction of a quark spin is surely not measurable, at least in practice, if not in theory. The possible directions, moreover, form a 2-parameter family, consistent with the property of colour confinement.

From a mathematical point of view, there is little prospect of linking the gauge group $S U(3)$ of quantum chromodynamics (QCD) as a theory of the strong force to a subgroup $S U(3)$ of the Clifford algebra. The alternatives therefore seem to be either to keep the gauge group outside the Clifford algebra completely, as in the standard model, or to try to re-write the formalism in terms of the 8-dimensional subalgebra $C \ell(0,3)$, generated by $\gamma_{1}, \gamma_{2}, \gamma_{3}$, rather than the 8-dimensional group $S U(3)$. I have no idea whether this is possible, and therefore leave this as an open problem. It seems to require a rather radical re-interpretation of the strong force as being not so much a force as a quantisation of space itself. Such an interpretation is certainly unconventional, but is perhaps hinted at by the phenomenon of asymptotic freedom $[18,28]$.

A more conventional approach would be to take the direct product of all the gauge groups and the spin group

$$
U(1) \times S U(2) \times S U(3) \times S L(2, \mathbb{C})
$$

and embed it in the real group

$$
S O(2) \times S O(4) \times S O(6) \times S L(4, \mathbb{R})
$$

so that there is an obvious 16 -dimensional real representation. Thus the group can be embedded in a Clifford algebra of an 8-dimensional space with signature $(8,0),(5,3),(4,4),(1,7)$ or $(0,8)$. A breaking of symmetry into $2+6$ and $4+4$ can be accommodated in $C \ell(4,3)$ or $C \ell(0,7)$. Alternatively, a breaking of symmetry to incorporate the complex structure can be accommodated in 
$C \ell(7,0), C \ell(5,2), C \ell(3,4)$ or $C \ell(1,6)$. All of these approaches, however, introduce a large number of extra parameters whose meanings and values still have to be explained.

\subsection{Further Remarks}

The suggested splitting of the 6 -vector representation as $3+1+2$ keeps $C \ell(0,3)$ intact, but splits $C \ell(3,0)$ into $C \ell(1,0)$ and $C \ell(2,0)$. A slightly closer match to the standard model splitting into $S U(3), U(1)$ and $S U(2)$ can be obtained by reversing the signatures, so that $C \ell(0,3)$ splits into $C \ell(0,1)$ and $C \ell(0,2)$, which are the complex numbers and quaternions, respectively. Hence we have obvious groups $U(1)$ and $S U(2)$, as subgroups of the group $\operatorname{Spin}(4)$ that lies inside $C \ell(0,3)$. The other half of the algebra is then $C \ell(3,0)$, the algebra of all $2 \times 2$ complex matrices, as a real 8 -dimensional algebra to replace the real 8-dimensional group $S U(3)$. The group that we actually get is, however, $G L(2, \mathbb{C})$, also 8 -dimensional as a real Lie group, with a normal subgroup $S L(2, \mathbb{C})$, that is isomorphic to, but distinct from, the relativistic spin group of Dirac. From a mathematical point of view, I cannot see any strong argument for adopting this reversal of signature, but it is possible there are good physical arguments for it.

It is also interesting to consider the Georgi-Glashow model [16] in this context. They unified $3+2$ into 5 , and because they were working with unitary groups, they used $S U(5)$ for unification of $S U(2)$ and $S U(3)$. But this group has dimension 24, and turned out to be too big, as it contains extra gauge bosons that cause proton decay, a phenomenon that has never been observed experimentally. If instead we unify Clifford algebras $C \ell(2,0)$ and $C \ell(0,3)$, then we obtain instead $C \ell(2,3)$, that is isomorphic to the Dirac algebra. The largest group that could possibly be relevant here is the general linear group $G L(4, \mathbb{C})$ of (complex) dimension 16 . This group is small enough to avoid the problem of proton decay, since it contains 13 dimensions for the standard 13 bosons, plus 3 dimensions for the spin group itself.

A final remark on chirality is in order. The suggestion to use $i \gamma_{5}$ as a generator for the electromagnetic $U(1)$, rather than $i$, is affected by the fact that $i \gamma_{5}$ anti-commutes with $\gamma_{\mu}$ for $\mu=0,1,2,3$. This implies that electromagnetism does not have a chirality, since the sign of $\gamma_{1} \gamma_{2} \gamma_{3}$ is not welldefined. On the other hand, the weak $S U(2)$ generated by $i, j, k$ commutes with these $\gamma_{\mu}$. This implies that the weak force does have a chirality, as is experimentally observed [48], since it distinguishes between $\gamma_{1} \gamma_{2} \gamma_{3}$ and its negative. Our choice of possible generators for the strong force algebra suggests that the strong force also should not have a chirality, since the $\gamma_{\mu}$ anti-commute with each other.

\subsection{Possible Relationships with Gravity}

At a philosophical level, the suggestion that the strong force is essentially describing the quantum structure of space relates closely to Einstein's approach $[8,9,36]$ to gravity, namely that gravity is not so much a force as a description of the shape of spacetime on a macroscopic scale. At a physical level, on the other hand, it seems much more likely to be the weak force 
that is responsible for curvature of spacetime, via the conversion of mass into neutrinos and antineutrinos. Perhaps the real story involves both, and might therefore require a new understanding of weak-strong mixing. At a mathematical level, the use of $C \ell(3,3)$ and the spin group $\operatorname{Spin}(3,3) \cong S L(4, \mathbb{R})$ may possibly be related to the use of $G L(4, \mathbb{R})$ in some modern approaches to general relativity $[19,21]$. But these superficial observations are a far cry from actually unifying the theories of general relativity and particle physics. A major stumbling block that I have already mentioned is the fact that it is mathematically impossible in this context to identify the group that is called the Lorentz group in particle physics with the group that is called the Lorentz group in general relativity.

It may therefore be impossible to build a unified theory on this basis. At the very least, one would have to contemplate the possibility that the relationship between these two 'Lorentz groups' should be downgraded from an equality to a mere analogy, as in the original work of Dirac. A first remark is that in $C \ell(3,3)$ there are many ways to find two copies of $S L(4, \mathbb{R})$ that commute with each other. Therefore one could in principle use one copy of $S L(4, \mathbb{R})$ for particle physics and the other for general relativity, simply placed side-by-side and not interacting with each other. Neither copy is equal to the spin group $\operatorname{Spin}(3,3)$, and neither of them respects the Clifford algebra structure, but they are obtained by suitable projections, defined by the pseudoscalar.

One could try to use the standard particle physics projections with $1 \pm \gamma_{5}$, but the discussion in this paper suggests that projections with $1 \pm \gamma_{0}$ are likely to be more useful in this context. This reflects the fact that general relativity describes a gravity that depends on total energy $\left(\gamma_{0}\right)$ rather than on rest mass $\left(\gamma_{5}\right)$. If one tries to use both pairs of projections together, one runs into problems because $\gamma_{0}$ and $\gamma_{5}$ do not commute with each other. Whatever choice of projections we make, the splitting of $C \ell(3,3)$ into two copies of $4 \times 4$ real matrices is a symmetry-breaking down to $C \ell(3,2)$. Further symmetrybreaking down to a single copy of $4 \times 4$ real matrices can be achieved by going to $C \ell(3,1)$ or $C \ell(2,2)$.

I have no idea whether it is actually possible to incorporate general relativity into $C \ell(3,3)$ in this way. If it is, then there is an overlap between the parts of the algebra that are used for particle physics and those that are used for gravity. Such an overlap is not part of current mainstream thinking, but has been considered at various times by Einstein [7], Sachs [31], Penrose [27] and others [43].

An alternative approach, more in line with mainstream ideas, is to go to a bigger Clifford algebra, so that this overlap can be avoided. For example, $C \ell(4,3)$ is the sum of two copies of $8 \times 8$ real matrices, which permits one copy to be used for $C \ell(3,3)$ to model particle physics, and the other to act on four dimensions of spacetime and four dimensions of energy-momentum, to describe classical physics and general relativity. Possibly it might be useful to go further, to $C \ell(4,4)$, in order to have enough room for the standard model strong gauge group $S U(3)$. 


\section{Conclusion}

In this paper, I have explored the subgroups of many small Clifford algebras in sufficient detail to conclude that $C \ell(3,3)$, on the generators $\gamma_{1}, \gamma_{2}, \gamma_{3}, \gamma_{5}$, $j \gamma_{5}$ and $k \gamma_{5}$, with pseudoscalar $\gamma_{0}$, is the closest match among the Clifford algebras to the algebraic foundations of the electro-weak part of the standard model of particle physics. I have shown that it incorporates the three generations of fermions, indexed by quaternions $i, j, k$, in the minimal possible way, consistent with the experimental properties of electrodynamics and the weak interaction.

The algebra $C \ell(3,3)$ also contains something that looks a bit like the strong force, but is implemented with the 8-dimensional Clifford algebra $C \ell(0,3)$, with generators $\gamma_{1}, \gamma_{2}, \gamma_{3}$, rather than the 8-dimensional Lie group $S U(3)$, which can only be implemented with a change of signature to $C \ell(0,6)$. This change of signature can be implemented within the real algebra, however, and does not require the complex algebra $\mathbb{C} \ell(6)$ that is used in other approaches to this problem. The main mathematical technique employed is to use the structures of the various Clifford algebras to describe symmetrybreaking within the underlying matrix algebras.

It is certainly not clear whether a model of this type can be used in place of the standard model formalism, since the embedding of the strong force is very different from its embedding in the standard model. Further work will be required to determine whether the proposals made here are consistent with experiment, since they are clearly inconsistent with the generally accepted theory. The justification for not rejecting the proposals immediately on these grounds lies in the potentially huge benefits if this difficulty can be overcome.

Not only are the three generations incorporated into the algebra in a natural rather than artificial manner, but so are all the unexplained parameters. If the model can be endowed with some actual numerical values that explain some of these parameters, or relationships between them, then there may be much to be gained from this approach. Some rather speculative ideas along these lines are presented in $[44,45]$, but without any particularly rigorous mathematical or physical justification.

Further unification with general relativity is hinted at by the coincidence of two groups, both isomorphic to $S L(4, \mathbb{R})$. This coincidence suggests the possibility of using either the Clifford algebra $C \ell(3,3)$ or perhaps a larger Clifford algebra such as $C \ell(4,3)$ or $C \ell(4,4)$ to include models of both particle physics and gravity. I make no attempt to build such a model, and again there may be good physical reasons why such a model cannot exist. But if such a model can be built, then there is a possibility that some gravitational parameters can be related to some parameters of particle physics in a way that current theories do not support. If so, then these relationships might, in principle at least, be exploited to explain things that the current models cannot explain.

More speculatively still, a theory of quantum gravity built on such a relationship might be able to explain the geometrical aspects of general relativity in terms of a more fundamental algebraic structure. Clifford algebras on 
their own, however, do not contain any obvious natural method of quantisation, or discretisation. For such purposes one needs at some stage to introduce finite groups into the algebra, whether this is done by reducing from a Lie group to its Weyl group, as in the standard model, or by some other process. One possibility that is rarely, if ever, considered, is to begin with a finite group, and build the Lie groups, Lie algebras, Clifford algebras and so on from them, rather than the other way round. This possibility is explored in various different ways in $[40-42,46]$.

Open Access. This article is licensed under a Creative Commons Attribution 4.0 International License, which permits use, sharing, adaptation, distribution and reproduction in any medium or format, as long as you give appropriate credit to the original author(s) and the source, provide a link to the Creative Commons licence, and indicate if changes were made. The images or other third party material in this article are included in the article's Creative Commons licence, unless indicated otherwise in a credit line to the material. If material is not included in the article's Creative Commons licence and your intended use is not permitted by statutory regulation or exceeds the permitted use, you will need to obtain permission directly from the copyright holder. To view a copy of this licence, visit http:// creativecommons.org/licenses/by/4.0/.

Publisher's Note Springer Nature remains neutral with regard to jurisdictional claims in published maps and institutional affiliations.

\section{References}

[1] Adamo, T.: Lectures on twistor theory. PoS Modave 2017, 003 (2018)

[2] Baez, J.C., Huerta, J.: The algebra of grand unified theories. Bull. Am. Math. Soc. 47, 483-552 (2010)

[3] Cabibbo, N.: Unitary symmetry and leptonic decays. Phys. Rev. Lett. 10(12), 531-533 (1963)

[4] Chester, D., Marrani, A., Rios, M.: Beyond the standard model with sixdimensional spacetime. arXiv:2002.02391 (2020)

[5] Coleman, S., Mandula, J.: All possible symmetries of the S matrix. Phys. Rev. 159(5), 1251 (1987)

[6] Dirac, P.A.M.: The quantum theory of the electron. Proc. R. Soc. A 117, 610 624 (1928)

[7] Einstein, A.: Spielen Gravitationsfelder im Aufbau der materiellen Elementarteilchen eine wesentliche Rolle?. Sitzungsberichte der Preussisschen Akad. d, Wissenschaften (1919)

[8] Einstein, A.: Die Grundlage der allgemeinen Relativitätstheorie. Ann. Phys. 49(7), 769-822 (1916)

[9] Einstein, A.: The Meaning of Relativity, 5th edn. Princeton University Press, Princeton (1955)

[10] Fairlie, D.B., Manogue, C.A.: Lorentz invariance and the composite string. Phys. Rev. D 34, 1832-1834 (1986)

[11] Feynman, R.P.: Quantum Electrodynamics. Westview Press, Boulder (1988) 
[12] Furey, C.: Generations: three prints, in colour. J. High Energy Phys. 10, 046 (2014)

[13] Furey, C.: Standard model physics from an algebra? $\mathrm{PhD}$ thesis. arXiv:1611.09182 (2016)

[14] Furey, N.: Three generations, two unbroken gauge symmetries, and one eightdimensional algebra. Phys. Lett. B 785, 84-89 (2018)

[15] Gell-Mann, M.: The eightfold way: a theory of strong interaction symmetry. Synchrotron Lab. Report CTSL-20, Cal. Tech. (1961)

[16] Georgi, H., Glashow, S.: Unity of all elementary-particle forces. Phys. Rev. Lett. 32(8), 438 (1974)

[17] Griffiths, D.: Introduction to Elementary Particles, 2nd edn. Wiley, New York (2008)

[18] Gross, D.J., Wilczek, F.: Ultraviolet behaviour of non-abelian gauge theories. Phys. Rev. Lett. 30(26), 1343-1346 (1973)

[19] Ivanenko, D., Sardanashvily, G.: The gauge treatment of gravity. Phys. Rep. 94, 1-45 (1983)

[20] Kobayashi, M., Maskawa, T.: CP-violation in the renormalizable theory of weak interaction. Progress Theoret. Phys. 49(2), 652-657 (1973)

[21] Leclerc, M.: The Higgs sector of gravitational gauge theories. Ann. Phys. 321, 708-743 (2006)

[22] Maki, Z., Nakagawa, M., Sakata, S.: Remarks on the unified model of elementary particles. Progress Theoret. Phys. 28(5), 870 (1962)

[23] Maldacena, J.M.: The Large $N$ limit of superconformal field theories and supergravity. Adv. Theor. Math. Math. 2, 231-252 (1998)

[24] Manogue, C.A., Dray, T.: Octonions, $E_{6}$, and particle physics. J. Phys. Conf. Ser. 254, 012005 (2010)

[25] Pati, J.C., Salam, A.: Lepton number as the fourth 'color'. Phys. Rev. D 10(1), 275-289 (1974)

[26] Penrose, R.: Twistor algebra. J. Math. Phys. 8(2), 345-366 (1967)

[27] Penrose, R.: The Road to Reality. Jonathan Cape, London (2004)

[28] Politzer, H.D.: Reliable perturbative results for strong interactions. Phys. Rev. Lett. 30(26), 1346-1349 (1973)

[29] Pontecorvo, B.: Inverse beta processes and non-conservation of lepton charge. Soviet Phys. JETP 7, 172 (1958)

[30] Porteous, I.R.: Clifford Algebras and the Classical Groups. Cambridge University Press, Cambridge (1995)

[31] Sachs, M.: Quantum Mechanics from General Relativity: An Approximation to a Theory of Inertia. D. Reidel Publishing Co., Cambridge (1986)

[32] Shirokov, D.S.: Symplectic, orthogonal and linear Lie groups in Clifford algebra. Adv. Appl. Clifford Algebras 25(3), 707-718 (2015)

[33] Shirokov, D.S.: On some Lie groups containing spin group in Clifford algebra. J. Geometry Symmetry Phys. 42, 73-94 (2016)

[34] Shirokov, D.S.: Classification of Lie algebras of specific type in complexified Clifford algebras. Linear Multilinear Algebra 66(9), 1870-1887 (2018)

[35] Stoica, O.C.: The standard model algebra-leptons, quarks, and gauge from the complex clifford algebra Cl6. Adv. Appl. Clifford Alg. 28, 52 (2018) 
[36] 't Hooft, G.: Introduction to General Relativity. Princeton, Rinton (2001)

[37] Todorov, I.: Superselection of the weak hypercharge and the algebra of the Standard Model. arXiv:2010.15621 (2020)

[38] van der Waerden, B.L.: Spinoranalyse. Nachr. Ges. Wiss. Göttingen Math.Phys. Ohne Angabe 20, 100-109 (1929)

[39] Weinberg, S.: A model of leptons. Phys. Rev. Lett. 19, 1264-66 (1967)

[40] Wilson, R.A.: A group-theorist's perspective on symmetry groups in physics. arXiv:2009.14613 (2020)

[41] Wilson, R.A.: Finite symmetry groups in physics. arXiv:2102.02817 (2021)

[42] Wilson, R.A.: Options for a finite group model of quantum mechanics. arXiv:2104.10165 (2021)

[43] Wilson, R.A.: Potential Uses of Representations of $S L(4, \mathbb{R})$ in Particle Physics, Preprint 19014. Isaac Newton Institute, Cambridge (2020)

[44] Wilson, R.A.: Remarks on the Group-Theoretical Foundations of Particle Physics, Preprint 19011. Isaac Newton Institute, Cambridge (2020)

[45] Wilson, R.A.: Possible Emergence of Fundamental Constants from a Generally Covariant Theory of Quantum Mechanics, Preprint 19013. Isaac Newton Institute, Cambridge (2020)

[46] Wilson, R.A.: Potential applications of modular representation theory to quantum mechanics. arXiv:2106.00550 (2021)

[47] Woit, P.: Quantum Theory, Groups and Representations. Springer, Berlin (2017)

[48] Wu, C.S., Ambler, E., Hayward, R.W., Hoppes, D.D., Hudson, R.P.: Experimental test of parity conservation in beta decay. Phys. Rev. 105(4), 1413-1415 (1957)

[49] Zee, A.: Group Theory in a Nutshell for Physicists. Princeton University Press, Princeton (2016)

Robert Arnott Wilson

Queen Mary University of London

London

UK

e-mail: r.a.wilson@qmul.ac.uk

Received: December 13, 2020.

Accepted: June 17, 2021. 